\title{
Pre-conception inter-pregnancy interval and risk of schizophrenia
}

Lihini Gunawardana,* George Davey Smith,* Stanley Zammit, Elise Whitley, David Gunnell, Sarah Lewis and Finn Rasmussen

\section{Summary}

It is hypothesised that the risk of schizophrenia may be elevated in children conceived following a short interpregnancy interval, when maternal folate stores are still being replenished. We examined the relationship between inter-pregnancy interval and schizophrenia risk in a Iongitudinal, population-based cohort. Risk of schizophrenia was increased by approximately $150 \%$ in those born following a pregnancy interval of $\leqslant 6$ months, but was not increased if the interval after birth of the participant, before conception of the subsequent sibling, was $\leqslant 6$ months. These findings support the hypothesis that folate (or other micronutrient) deficiency during fetal development may be an important risk factor for schizophrenia.

\section{Declaration of interest}

None.

the post-birth inter-pregnancy interval, defined as the time between the index birth and conception of the subsequent live birth. The hypothesis regarding folate depletion would apply only to the pre-birth interval. We controlled for gender, calendar year, parental age, parental socioeconomic status, place of birth, obstetric complications (Caesarean section or uterine atony), birthweight, length of gestation, history of psychosis in parents or siblings, family size, and birth order. First-borns were excluded from analyses of pre-birth inter-pregnancy interval (167767 excluded leaving 183921) and last-borns from analyses of post-birth inter-pregnancy interval (168 831 excluded leaving 182 857).

All statistical analysis was performed with Stata Release 11.0 for Windows. We used Cox's proportional hazards models to assess the influence of inter-pregnancy interval on schizophrenia before and after adjustment for confounders. People were censored at whichever of the following occurred first: time of first admission for schizophrenia, death, emigration or 31 December 2002. We used the cluster option in Stata to adjust standard errors for clustering of cases within families. We tested the validity of the proportional hazards assumption graphically.

\section{Results}

The cohort comprised 777832 people born in Sweden (19731980 ) and still resident there at age 16. Information was obtained from linkage between the medical birth registry, the population and housing census (1990) and the cause of death and emigration registers. Diagnoses of schizophrenia (ICD-10 ${ }^{11}$ (1997-2002) code F20; ICD-9 $9^{12}$ (1987-1997) code 295, except 295F and 295H) were identified through linkage with the in-patient discharge register (up to 31 December 2002). For the purposes of the current analysis, 13258 (1.7\%) multiple births and 401623 (51.6\%) only children were excluded, leaving a total of 362951 individuals. Of these, 11263 (3.1\%) were excluded because of missing data.

The pre-birth inter-pregnancy interval was defined as the amount of time between the date of delivery for the immediately prior pregnancy and the date of conception (approximated on the basis of date of delivery and gestational age) for the index pregnancy, and categorised as follows: $\leqslant 6$ months; $7-12$ months, 13-24 months, 25-36 months and $>37$ months. The baseline group was the 13-24 months inter-pregnancy interval, as it may take up to 1 year for maternal folate levels to return to pre-pregnancy levels. ${ }^{8}$ We also examined the association with

*These authors contributed equally to the work.
Of the 183921 people included in the final analysis, 164 were diagnosed with schizophrenia. Schizophrenia rates varied with the preceding birth interval (Table 1). Those born with shorter inter-pregnancy intervals had a higher risk of schizophrenia. The hazard ratio for developing schizophrenia if the inter-pregnancy interval prior to conception was $<6$ months $v$. 13-24 months was 3.29 (95\% CI 2.00-5.41, $P<0.001$ ), and 1.96 (95\% CI $1.27-$ 3.05) when the inter-pregnancy interval was $7-12$ months.

Adjusting for gestational age, birth order, paternal age and obstetric complications made no difference to the results and these factors were excluded from the final model. Children born after shorter inter-pregnancy intervals tended to be from larger families, be born in earlier calendar years, have younger parents with lower income and socioeconomic position, and were more likely to have a family history of psychosis (online Table DS1). Adjusting for these variables attenuated the associations observed. The adjusted hazard ratio for pre-birth inter-pregnancy interval $<6$ months was $2.62(95 \%$ CI $1.5-4.57, P<0.001)$ and 1.78 (95\% CI 1.14-2.80) when the interval was $7-12$ months.

This association between inter-pregnancy interval and schizophrenia risk was not apparent for the post-birth interval. 


\begin{tabular}{|c|c|c|c|c|c|c|}
\hline & Participants, $n$ & Cases, $n(\%)$ & Unadjusted HR (95\% Cl) & $P$ & Adjusted $\mathrm{HR}^{\mathrm{a}}(95 \% \mathrm{Cl})$ & $P$ \\
\hline \multicolumn{7}{|c|}{ Pre-birth interval, months } \\
\hline$\leqslant 6$ & 9589 & $25(0.26)$ & $3.29(2.00-5.41)$ & & $2.62(1.50-4.57)$ & \\
\hline $7-12$ & 22279 & $34(0.15)$ & $1.96(1.27-3.05)$ & & $1.78(1.14-2.80)$ & \\
\hline $13-24$ & 61535 & $47(0.08)$ & 1.00 & & 1.00 & \\
\hline $25-36$ & 48726 & $31(0.06)$ & $0.87(0.55-1.36)$ & & $0.90(0.57-1.06)$ & \\
\hline $37+$ & 41792 & $27(0.06)$ & $0.95(0.59-1.53)$ & & $1.01(0.61-1.65)$ & \\
\hline All intervals (trend) & 183921 & $164(0.09)$ & & $<0.001$ & & 0.002 \\
\hline \multicolumn{7}{|c|}{ Post-birth interval, months } \\
\hline$\leqslant 6$ & 8958 & $12(0.13)$ & $0.97(0.51-1.85)$ & & $0.73(0.38-1.40)$ & \\
\hline $7-12$ & 21872 & $33(0.15)$ & $1.08(0.73-1.62)$ & & $0.96(0.63-1.44)$ & \\
\hline $13-24$ & 61369 & $89(0.15)$ & 1.00 & & 1.00 & \\
\hline $25-36$ & 48803 & $53(0.11)$ & $0.72(0.51-1.01)$ & & $0.75(0.53-1.06)$ & \\
\hline $37+$ & 41855 & $60(0.14)$ & $0.90(0.64-1.25)$ & & $0.89(0.63-1.25)$ & \\
\hline All intervals (trend) & 182857 & $247(0.14)$ & & 0.22 & & 0.72 \\
\hline
\end{tabular}

The adjusted odds ratio if the post-birth interval was $<6$ months was 0.73 (95\% CI $0.38-1.40, P=0.28)$.

\section{Discussion}

Individuals conceived within 1 year, and especially within 6 months of the date of delivery for the preceding pregnancy, had an increased risk of developing schizophrenia compared with longer inter-pregnancy intervals.

Women who conceive following a short inter-pregnancy interval differ on a number of characteristics that could influence risk of schizophrenia, compared with women with longer intervals. Adjustment for confounders attenuated the associations we observed by approximately $30 \%$. Although residual confounding remains possible, the pattern of increasing risk of schizophrenia in those with short pre-birth inter-pregnancy intervals was not observed in those with short post-birth intervals. This argues against residual confounding being a satisfactory explanation for our findings, and lends support to the hypothesis that pre-conception maternal folate reserves may play a causal role in the aetiology of schizophrenia.

Our findings are consistent with previous research in this area, ${ }^{10}$ while shorter inter-pregnancy intervals have also been associated with autism ${ }^{13}$ and other adverse outcomes including neural tube defects. ${ }^{3,4}$ The period immediately following the birth of a preceding pregnancy may be a particular period of risk where depleted maternal nutrient reserves could affect neurogenesis during early fetal development. For example, folate deficiency affects DNA synthesis, repair and methylation, and can alter expression of genes that regulate neurodevelopment. ${ }^{5}$ In keeping with this, raised homocysteine levels that occur secondary to folate deficiency and polymorphisms in the MTHFR gene that influences folate metabolism have both been associated with schizophrenia. ${ }^{6}$

Alternative explanations for our findings include depletion of other micronutrients (e.g. iron or vitamin D deficiency ${ }^{14,15}$ ), and increased maternal stress levels that might be greater for shorter prior pregnancy intervals. ${ }^{9}$ Further study in this field, for example of long-term effects of specific micronutrient depletion on neurodevelopment in animal models, may provide important insights into the aetiology of schizophrenia.

If micronutrient deficiency is established as the causal mechanism for our findings, this would support the promotion of dietary supplements in the postpartum period as well as periconceptually, ${ }^{3}$ particularly in low- and middle-income countries where shorter inter-pregnancy intervals are more common.

\section{Funding}

S.Z. funded through a Clinician Scientist Award from the National Assembly for Wales.

Lihini Gunawardana, MRCPsych, MRC Centre for Neuropsychiatric Genetics and Genomics, Cardiff University, UK; George Davey Smith, MD, DSC, School of Social and Community Medicine, University of Bristol, UK; Stanley Zammit, PhD, MRC Centre for Neuropsychiatric Genetics and Genomics, Cardiff University, and School of Social and Community Medicine, University of Bristol, UK; Elise Whitley, PhD, David Gunnell, FFPH, DSC, Sarah Lewis, BSC, PhD, School of Social and Community Medicine, University of Bristol, UK; Finn Rasmussen, MD, MPH, PhD, Department of Public Health Sciences, Karolinska Institute, Stockholm, Sweden

Correspondence: Dr Stanley Zammit, Department of Psychological Medicine \& Neurology, Cardiff University, Heath Park, Cardiff CF14 4XN, UK. Email: zammits@cardiff.ac.uk

First received 8 Feb 2011, final revision 18 May 2011, accepted 16 of Jun 2011

\section{References}

1 Susser ES, Lin SP. Schizophrenia after prenatal exposure to the Dutch Hunger Winter of 1944-1945. Arch Gen Psychiatry 1992; 49: 983-8.

2 St Clair D, Xu M, Wang P, Yu Y, Fang Y, Zhang F, et al. Rates of adult schizophrenia following prenatal exposure to the Chinese famine of 1959-1961. JAMA 2005; 294: 557-62.

3 Smits LJ, Essed GG. Short interpregnancy intervals and unfavourable pregnancy outcome: role of folate depletion. Lancet 2001; 358: 2074-7.

4 Todoroff K, Shaw GM. Prior spontaneous abortion, prior elective termination, interpregnancy interval, and risk of neural tube defects. Am J Epidemiol 2000; 151: 505-11.

5 Brown AS, Susser ES. Prenatal nutrinional deficiency and risk of adult schizophrenia. Schizophr Bull 2008; 34: 1054-63.

6 Zammit S, Lewis S, Gunnell D, Davey Smith G. Schizophrenia and neural tube defects: comparisons from an epidemiological perspective. Schizophr Bull 2007; 33: 853-8.

7 Milman N, Byg KE, Hvas AM, Bergholt T, Eriksen L. Erythrocyte folate, plasma folate and plasma homocysteine during normal pregnancy and postpartum: a longitudinal study comprising 404 Danish women. Eur J Haematol 2006; 76 : 200-5.

8 O'Rourke KM, Redlinger TE, Waller DK. Declining levels of erythrocyte folate during the postpartum period among Hispanic women living on the TexasMexico border. J Womens Health Gend Based Med 2000; 9: 397-403.

9 Dewey KG, Cohen RJ. Does birth spacing affect maternal or child nutritional status? A systematic literature review. Matern Child Nutr 2007; 3: 151-73.

10 Smits L, Pedersen C, Mortensen P, van Os J. Association between short birth intervals and schizophrenia in the offspring. Schizophr Res 2004; 70: 49-56.

11 World Health Organization. International Statistical Classification of Diseases and Related Health Problems, 10th Revision. WHO, 1992.

12 World Health Organization. International Classification of Diseases, Ninth Revision. WHO, 1977.

13 Cheslack-Postava K, Liu K, Bearman PS. Closely spaced pregnancies are associated with increased odds of autism in california sibling births. Pediatrics 2011; 127: 246-53.

14 Insel BJ, Schaefer CA, McKeague IW, Susser ES, Brown AS. Maternal iron deficiency and the risk of schizophrenia in offspring. Arch Gen Psychiatry 2008; 65: 1136-44.

15 McGrath JJ, Eyles DW, Pedersen CB, Anderson C, Ko P, Burne TH, et al. Neonatal vitamin $D$ status and risk of schizophrenia: a population-based case-control study. Arch Gen Psychiatry 2010; 67: 889-94. 